\title{
A Theorem on Alternants
}

By D. E. Littlewood (University College, Swansea).

Communicated by H. W. Turnboll.

(Received 17th January, 1936. Read 7th February, 1936.)

The following is a direct proof of a theorem by Zia-ud-Din ${ }^{1}$.

Let $\{\nu\} \equiv\left\{\nu_{1}, \nu_{2}, \ldots, \nu_{p}\right\}$ be any $S$-function ${ }^{2}$ of weight $r+s$ such that

$$
\{\nu\} \Delta\left(x_{1}, x_{2}, \ldots, x_{p}\right)=\Sigma \pm x_{1}^{\nu_{1}+p-1} x_{2}^{\nu_{2}+p-2} \ldots x_{p}^{\nu_{\rho}}
$$

in the alternant denoted in the theorem by $A(\alpha \beta \gamma \ldots)$. Let $\{\mu\}$ be an $S$-function of weight $s$, equal to $h\left(\begin{array}{c}0 p q \ldots . \\ 012 \ldots\end{array}\right)$, and let $\{\lambda\}$ be an $S$-function of weight $r$, such that $\{\lambda\} \Delta\left(x_{1}, \ldots, x_{p}\right)$ is obtained with coefficient $g_{\lambda \mu \nu}$ by diminishing the indices in the alternant $\{\nu\} \Delta\left(x_{1}, \ldots, x_{p}\right)$ according to the theorem.

Obviously, by direct multiplication, $g_{\lambda \mu \nu}$ is the coefficient of $\{\nu\} \Delta\left(x_{1}, \ldots, x_{p}\right)$ in the product of $\{\lambda\} \Delta\left(x_{1}, \ldots, x_{p}\right)$ and $\{\mu\}$, i.e., the coefficient of $\{\nu\}$ in the product $\{\lambda\}\{\mu\}$.

If we now proceed to the associated $S$-functions, denoting these by a bar, clearly $g_{\lambda \mu \nu}$ is also the coefficient of $\{\nu\}$ in $\{\lambda\}\{\mu\}$. This proves the theorem.

1 Proc. Edinburgh Math. Soc., 4 (1934), 51.

2 D. E. Littlewood and A. R. Richardson, Phil. Trans. Roy. Soc. (A), 233 (1934), 99. 